Bull. Austral. Math. Soc.

$05 \mathrm{~A} 15,05 \mathrm{~A} 19$

VOL. 58 (1998) [359-371]

\title{
GENERALISATION OF EULER'S IDENTITY
}

\section{A. Sofo And P. Cerone}

An investigation of representation of Abel type infinite sums in closed form will be presented in this paper. An arbitrary order forced differential-difference equation will be analysed from which sums will be generated. Identities of resulting infinite sums will be proved.

\section{INTRODUCTION}

This paper is concerned with the problem of generalising the identity

$$
\sum_{n=0}^{\infty} \frac{b^{n}}{n !} e^{-b(t-a n)}(t-a n)^{n}=\frac{1}{1+a b} .
$$

In a paper written in Latin, and published in 1779, Euler [7] (see also [8]) began with a Lambert series and through various manipulations derived the identity

$$
\sum_{n=0}^{\infty} \frac{(s+n)^{n}}{n !}\left(\frac{\log x}{x}\right)^{n}=\frac{x^{s}}{1-\log x}
$$

and if we put $s a=-t$ and $x=e^{-a b}$ then (1.2) is identical to (1.1). Similarly, in a paper written in French and published in 1902, Jensen [12] substituted two exponential functions into an alternate form of Lagrange's theorem and obtained the identity

$$
\sum_{n=0}^{\infty} \frac{(\alpha+\beta n)^{n}}{n !}\left(z e^{-\beta z}\right)^{n}=\frac{e^{\alpha z}}{1-\beta z}
$$

and on putting $z=b, \beta=-a$ and $\alpha=t$ then (1.3) becomes (1.1). Ramanujan was also interested in a modification of (1.1) and it is noted in a question given in his collected papers which are edited by Hardy, Seshu Aiyar and Wilson [11] and, finally Berndt [2] gives an excellent, short historical account of a modification of (1.1).

The occurence of (1.1), in many guises, is quite prolific in a range of different branches of applications. A modification of the sum (1.1) appears as a problem in the work of Pólya and Szegö [13], and it appears in the works of Bruiwer [3] and [4], in his

Received 11th February, 1998

Copyright Clearance Centre, Inc. Serial-fee code: 0004-9729/98 $\$ A 2.00+0.00$. 
study of differential-difference equations. In the analysis of the delay in the answering of telephone calls Erlang [6] obtains an integro-differential-difference equation from which the sum (1.1) may be extracted. The sum (1.1) also arises in the works of Feller [9] on ruin problems, Hall [10] on coverage processes, Smith [15] on renewal theory and Tijms [17] on stochastic modelling. The authors can also extract the sum (1.1) from models of grazing systems [18] and other areas as detailed by Cerone and Sofo [5].

The layout of this paper will be as follows. In Section 2 we develop our method, which is different from that given by Euler or Jensen, for generating (1.1) We consider a forced differential-difference equation of arbitrary order, and by the use of Laplace transform techniques generate our required sum. The required identity will be completed by using residue theory and an appeal to a Tauberian theorem. The identity, it will be shown, depends on a dominant zero of an associated characteristic function. In Section 3 we develop a recurrence relation for use in determining specific closed form expressions of the infinite sum, and in Section 4 a proof of the main theorem in this paper will be given, together with a functional relationship. In Section 5 we indicate an extension to our main result.

\section{Methodology}

Consider, for a well behaved function $f(t)$, the forced dynamical system with constant real coefficients $b$ and $c$, real delay parameter $a$, and all initial conditions at rest,

$$
\left.\begin{array}{cc}
\sum_{n=0}^{R}\left(\begin{array}{c}
R \\
R-n
\end{array}\right) c^{R-n} \sum_{r=0}^{n}\left(\begin{array}{l}
n \\
r
\end{array}\right) b^{n-r} f^{(r)}(t-(R-n) a)=w(t) ; & t>R a \\
\sum_{r=0}^{R}\left(\begin{array}{l}
R \\
r
\end{array}\right) b^{R-r} f^{(r)}(t)=w(t) ; & 0<t \leqslant R a .
\end{array}\right\}
$$

In the system (2.1) $w(t)$ is a forcing term, $t$ a real variable, and $R$ is positive integer, being the order of the differential-delay equation. A method of analysing the solution of system (2.1) is by the use of Laplace transform techniques. Taking the Laplace transform of (2.1) and utilising the property

$$
\mathcal{L}\left(f^{(n)}(t-k)\right)=e^{-k p}\left(p^{n} F(p)-\sum_{j=1}^{n} p^{n-j} f^{(j-1)}(0)\right),
$$

we obtain

$$
\left(\sum_{j=0}^{R}\left(\begin{array}{c}
R \\
j
\end{array}\right)(p+b)^{j}\left(c e^{-a p}\right)^{R-j}\right) F(p)=W(p) .
$$


From (2.2)

$$
F(p)=\frac{W(p)}{\left(p+b+c e^{-a p}\right)^{R}}
$$

where $F(p)$ and $W(p)$ are the Laplace transforms of $f(t)$ and $w(t)$ respectively. Equation (2.3) may be expanded in series so that

$$
\begin{aligned}
F(p) & =\frac{W(p)}{(p+b)^{R}\left(1+\frac{c e^{-a p}}{p+b}\right)^{R}} \\
& =\sum_{n=0}^{\infty}\left(\begin{array}{c}
n+R-1 \\
n
\end{array}\right) \frac{W(p)\left(-c e^{-a p}\right)^{n}}{(p+b)^{n+R}} .
\end{aligned}
$$

To bring out the essential features of our results we may choose the forcing term $w(t)=$ $\delta(t)$, the Dirac delta function, such that $W(p)=1$. Substituting $W(p)$ into $(2.4)$ and taking the inverse Laplace transform, we have

$$
f(t)=\sum_{n=0}^{\infty}\left(\begin{array}{c}
n+R-1 \\
n
\end{array}\right) \frac{(-c)^{n} e^{-b(t-a n)}(t-a n)^{n+R-1}}{(n+R-1) !} H(t-a n),
$$

where $H(x)$ is the unit Heaviside step function. The inverse of (2.3), a solution of the system (2.1), by Laplace transform theory may also be written as

$$
f(t)=\frac{1}{2 \pi i} \int_{\gamma-i \infty}^{\gamma+i \infty} e^{p t} F(p) d p
$$

for an appropriate choice of $\gamma$ such that all the zeros of the characteristic function

$$
g_{1}(p)=p+b+c e^{-a p}
$$

are contained to the left of the line in the Bromwich contour, and $F(P)$ is defined by (2.3). Now by the residue theorem

$$
f(t)=\sum \text { residues of }\left(e^{p t} F(p)\right)
$$

which suggests that solution of $f(t)$ may be written in the form

$$
f(t)=\sum_{r} Q_{r} e^{p_{r} t}
$$


where the sum is over all the characteristic zeros $p_{r}$ of $g_{1}(p)=0$ and $Q_{r}$ is the contribution of the residues in $F(p)$ at $p=p_{r}$. It was shown by Cerone and Sofo [5] that the zeros of the characteristic function (2.6) with restriction

$$
\left|a c e^{1+a b}\right|<1
$$

are all distinct. The poles of the expression (2.3) depend on the zeros of the characteristic function (2.6), namely, the zeros of $g_{1}(p)=0$. The dominant distinct zero, $p_{0}$, of $g_{1}\left(p_{0}\right)=0$ is defined as the one with the greatest real part and therefore by a Tauberian theorem [1] we have that asymptotically

$$
f(t) \sim \sum_{k=0}^{R-1} Q_{R, k}\left(p_{0}\right) \frac{t^{R-k-1} e^{p_{0} t}}{(R-k-1) !}
$$

From (2.5) and (2.8), we have that

$$
f(t)=\sum_{n=0}^{[t / a]}\left(\begin{array}{c}
n+R-1 \\
n
\end{array}\right) \frac{(-c)^{n} e^{-b(t-a n)}(t-a n)^{n+R-1}}{(n+R-1) !} \sim \sum_{k=0}^{R-1} Q_{R, k}\left(p_{0}\right) \frac{t^{R-k-1} e^{p_{0} t}}{(R-k-1) !}
$$

where $[x]$ represents the integer part of $x$ and the residue contribution, $Q_{R, k}\left(p_{0}\right)$, is given by

$$
k ! Q_{R, k}\left(p_{0}\right)=\lim _{p \rightarrow p_{0}}\left[\frac{d^{k}}{d p^{k}}\left(\left(p-p_{0}\right)^{R} F(p)\right)\right] ; \quad k=0,1,2,3, \ldots,(R-1),
$$

since (2.3) has a pole of order $R$ at the distinct dominant zero, $p=p_{0}$, for $1-a c \neq 0$. From now on we may take, without any loss of generality, $b+c=0$ and $1+a b \neq 0$. These conditions simply allow the distinct dominant zero, $p_{0}$, of the characteristic function (2.6), with restriction (2.7), to occur at $p_{0}=0$, and therefore from (2.6) and (2.10) respectively

$$
g(p)=p+b-b e^{-a p}
$$

and

$$
k ! Q_{R, k}(0)=\lim _{p \rightarrow 0}\left[\frac{d^{k}}{d p^{k}}\left(\left(\frac{p}{g(p)}\right)^{R}\right)\right] ; \quad k=0,1,2,3, \ldots,(R-1) .
$$

ThEOREM 2.1. Let

$$
T_{n}(b, R, a, t)=\left(\begin{array}{c}
n+R-1 \\
n
\end{array}\right) \frac{b^{n} e^{-b(t-a n)}(t-a n)^{n+R-1}}{(n+R-1) !}
$$

and

$$
S_{R}(b, a, t)=\sum_{n=0}^{\infty} T_{n}(b, R, a, t)
$$


which is convergent for all values of $b, R, a$ and $t$ in the region of convergence (2.7). Then

$$
S_{R}(b ; a, t)=\sum_{k=0}^{R-1} Q_{R, k}(0) \frac{t^{R-k-1}}{(R-k-1) !} .
$$

The series (2.14) is known as an Abel type series, because of the $(t-a n)^{n+R-1}$ term, and the convergence region (2.7) may be obtained by applying the ratio test to the term $T_{n}(b, R, a, t)$ in (2.13). A proof of the main Theorem (2.1) will follow shortly. Firstly we shall develop a useful recurrence relation for the evaluation of the terms $Q_{R, k}(0)$ in (2.12) and an identity for the $Q_{R, k}(0)$ terms. Secondly, using the terms $Q_{R, k}(0)$ we shall give some closed form representations of the infinite sum (2.15). Thirdly, a recurrence relation for the series (2.14) will be developed, and finally an induction argument on the integer $R$ will be applied to prove the main theorem (2.1).

\section{RECURRENCES AND CLOSED FORMS}

LEMmA 3.1. A recurrence relation for the evaluation of the terms $Q_{R, k}(0)$ in (2.12) is

$$
(k+1) Q_{R, k+1}(0)=R \sum_{\mu=0}^{k} \frac{(-1)^{\mu} b a^{\mu+2}(\mu+1)}{(\mu+2) !} Q_{R+1, k-\mu}(0)
$$

with

$$
Q_{R, 0}(0)=\frac{1}{(1+a b)^{R}}
$$

Proof: From (2.12)

$$
Q_{R, 0}(0)=\lim _{p \rightarrow 0}\left[\left(\frac{p}{g(p)}\right)^{R}\right]=\frac{1}{(1+a b)^{R}}
$$

Also from (2.12)

$$
\begin{aligned}
(k+1) ! Q_{R, k+1}(0) & =\lim _{p \rightarrow 0}\left[\frac{d^{k}}{d p^{k}}\left\{\frac{d}{d p}\left(\frac{p}{g(p)}\right)^{R}\right\}\right] ; \quad k=0,1,2, \ldots,(R-2) \\
& =R \lim _{p \rightarrow 0}\left[\frac{d^{k}}{d p^{k}}\left\{\frac{p^{R-1}\left(g(p)-p g^{\prime}(p)\right)}{(g(p))^{R+1}}\right\}\right]
\end{aligned}
$$

where $g(p)$ is defined in (2.11) and its first derivative is

$$
g^{\prime}(p)=1+a b e^{-a p} .
$$


Letting $h(p)=g(p)-p g^{\prime}(p)$ we find that $h(0)=0$ and $h^{\prime}(0)=0$ and therefore on expanding $h(p)$ as a Taylor series about $p=0$, we may write, from (3.2)

$$
(k+1) ! Q_{R, k+1}(0)=R \lim _{p \rightarrow 0}\left[\frac{d^{k}}{d p^{k}}\left\{\left(\frac{p}{g(p)}\right)^{R+1} \frac{h(p)}{p^{2}}\right\}\right]
$$

where

$$
\frac{h(p)}{p^{2}}=\sum_{j=2}^{\infty} \frac{(-a)^{j} b p^{j-2}(j-1)}{j !}=B(p)
$$

Hence from (3.3)

$$
\begin{aligned}
(k+1) ! Q_{R, k+1}(0) & =R \lim _{p \rightarrow 0}\left[\frac{d^{k}}{d p^{k}}\left\{\left(\frac{p}{g(p)}\right)^{R+1} B(p)\right\}\right] \\
& =R \lim _{p \rightarrow 0}\left[\sum_{\mu=0}^{k}\left(\begin{array}{l}
k \\
\mu
\end{array}\right)\left\{\left(\frac{p}{g(p)}\right)^{R+1}\right\}^{(k-\mu)} B^{(\mu)}(p)\right]
\end{aligned}
$$

by the Leibniz rule of differentiation, where

$$
B^{(\mu)}(p)=\frac{d^{\mu}}{d p^{\mu}} B(p)
$$

Now since

$$
\lim _{p \rightarrow 0}\left[\frac{d^{\mu}}{d p^{\mu}} B(p)\right]=\frac{(-1)^{\mu} b a^{\mu+2}}{(\mu+2)}
$$

and substituting in (3.4) we find that

$$
(k+1) Q_{R, k+1}(0)=R \sum_{\mu=0}^{k} \frac{(-1)^{\mu} b a^{\mu+2}(\mu+1)}{(\mu+2) !} Q_{R+1, k-\mu}(0)
$$

which completes the proof of Lemma 3.1.

Now using equation (3.1) we can list some values of $Q_{R, k}(0)$ as given in Table 1.

The following lemma will be useful in the proof of the main theorem.

LEMMA 3.2 .

$$
\begin{aligned}
R(1+a b) Q_{R+1, k+1}(0)+a b \frac{d}{d b} Q_{R, k}(0)=(R-(k+1)) Q_{R, k+1}(0) & \\
k & =0,1,2,3, \ldots,(R-1) .
\end{aligned}
$$

Proof: From (2.12)

$$
\frac{d}{d b}\left\{k ! Q_{R, k}(0)\right\}=\frac{d}{d b}\left\{\lim _{p \rightarrow 0}\left[\frac{d^{k}}{d p^{k}}\left(\frac{p}{g(p)}\right)^{R}\right]\right\}
$$




\begin{tabular}{l|c}
$k$ & $k ! Q_{R, k}(0)$ \\
\hline 0. & $\frac{1}{(1+a b)^{R}}$ \\
1. & $\frac{R b a^{2}}{2(1+a b)^{R+1}}$ \\
2. & $\frac{R b a^{3}[a b(3 R-1)-4]}{12(1+a b)^{R+2}}$ \\
3. & $\frac{R b a^{4}\left[2-4 a b R+a^{2} b^{2} R(R-1)\right]}{8(1+a b)^{R+3}}$ \\
4. & $\frac{R b a^{5}\left[a^{3} b^{3}\left(15 R^{3}-30 R^{2}+5 R+2\right)-a^{2} b^{2}\left(120 R^{2}+40 R-16\right)+a b(200 R+56)-48\right]}{240(1+a b)^{R+4}}$ \\
5. & $R b a^{6}\left[a^{4} b^{4}\left(3 R^{4}-10 R^{3}+5 R^{2}+2 R\right)-a^{3} b^{3}\left(40 R^{3}-40 R^{2}-16 R\right)\right.$ \\
& $\frac{\left.+a^{2} b^{2}\left(140 R^{2}+36 R-8\right)-a b(128 R+64)+16\right]}{96(1+a b)^{R+5}}$
\end{tabular}

Table 1: Values of $Q_{R, k}(0)$ for $k=0,1,2,3,4$ and 5.

Interchanging the order of differentiation in the second term, and after some simplification, we obtain

$$
\frac{d}{d b}\left\{k ! Q_{R, k}(0)\right\}=\lim _{p \rightarrow 0}\left[\frac{d^{k}}{d p^{k}}\left\{\frac{-R p^{R} \frac{d}{d b} g(p)}{g^{R+1}(p)}\right\}\right]
$$

and since

$$
\frac{d}{d b} g(p)=\frac{g(p)-p}{b}
$$

we have

$$
k ! \frac{d}{d b} Q_{R, k}(0)=-\frac{R}{b} \lim _{p \rightarrow 0}\left[\frac{d^{k}}{d p^{k}}\left\{\frac{p^{R}}{g^{R}(p)}-\frac{p^{R+1}}{g^{R+1}(p)}\right\}\right],
$$

and using (2.12)

$$
b \frac{d}{d b} Q_{R, k}(0)=R\left(Q_{R+1, k}(0)-Q_{R, k}(0)\right)
$$

Now, the $Q(0)$ terms may be associated by constants $c_{1}, c_{2}$, and $c_{3}$ such that

$$
Q_{R+1, k+1}(0)+c_{1}(R-(k+1)) Q_{R, k+1}(0)+c_{2} Q_{R, k}(0)+c_{3} Q_{R+1, k}(0)=0
$$


the $(R-(k+1))$ factor in $Q_{R, k+1}(0)$ is required since it does not contribute for $R=$ $k+1$. From Table 1 we choose three $k$ values and substitute the respective $Q(0)$ values in (3.7). Then solving for $c_{1}, c_{2}$, and $c_{3}$ we obtain

$$
c_{1}=-\frac{1}{R(1+a b)}, \quad c_{2}=-\frac{a}{1+a b}, \quad \text { and } c_{3}=\frac{a}{1+a b} .
$$

Hence from (3.7)

$$
Q_{R+1, k+1}(0)-\frac{(R-(k+1))}{R(1+a b)} Q_{R, k+1}(0)-\frac{a}{1+a b} Q_{R, k}(0)+\frac{a}{1+a b} Q_{R+1, k}(0)=0 .
$$

By substituting (3.6) in (3.8), and after some minor manipulation, we obtain the result (3.5), and the proof of Lemma 3.2 is complete.

Using the $Q_{R, k}(0)$ in Table 1 , some closed form representation of the infinite series (2.15) are listed below.

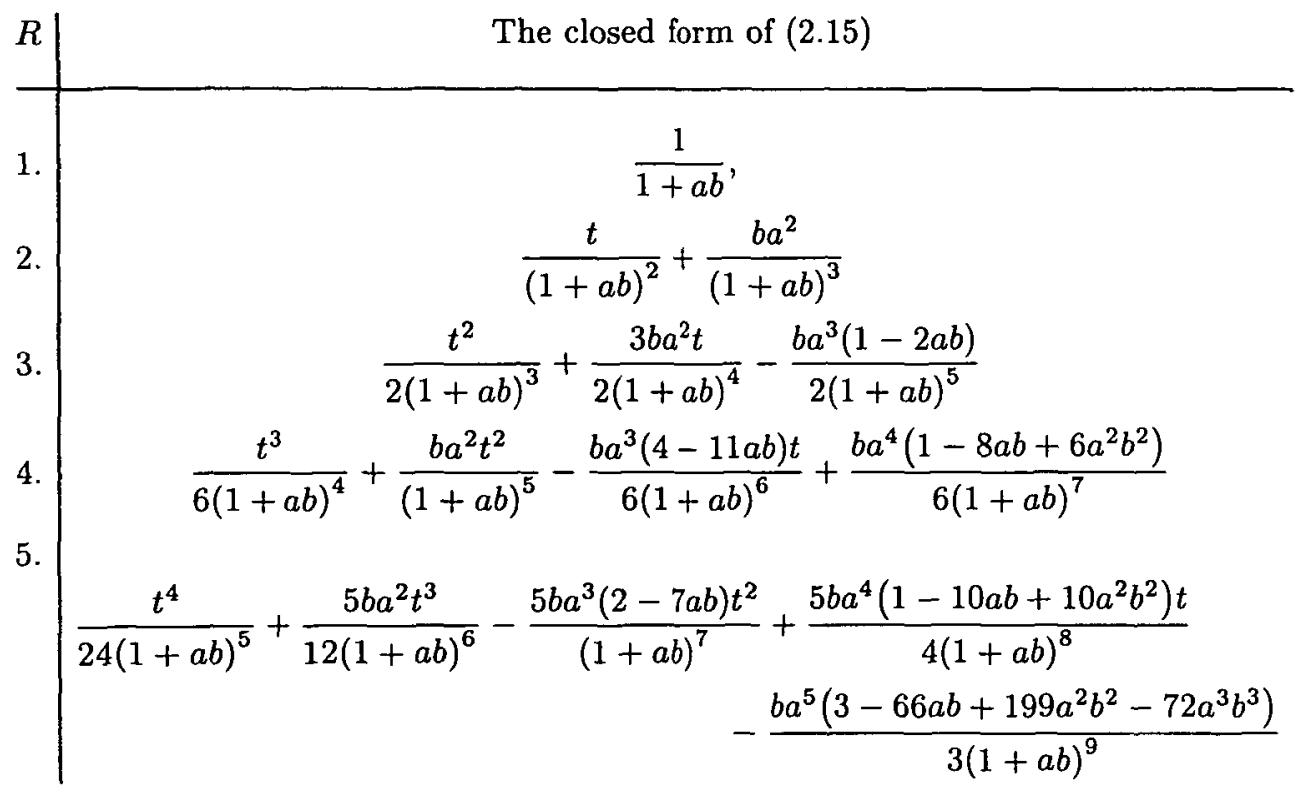

Table 2. The closed form expression of (2.15) for $R=1,2,3,4$ and 5 .

In the next section we give a proof of the main Theorem 2.1.

\section{LemMA AND PROOF OF THEOREM 2.1}

The following lemma will be useful for the proof of Theorem 2.1. 
LEMMA 4.1. A recurrence relation for the infinite series (2.14) is

$$
R(1+a b) S_{R+1}+a b \frac{d}{d b} S_{R}-t S_{R}=0 .
$$

Proof: From (2.13) and (2.14)

$$
S_{R+1}=\sum_{n=0}^{\infty}\left(\begin{array}{c}
n+R \\
n
\end{array}\right) \frac{b^{n} e^{-b(t-a n)}(t-a n)^{n+R}}{(n+R) !}=\frac{1}{R}\left(t S_{R}-a \sum_{n=0}^{\infty} n T_{n}\right) .
$$

Also, from (2.13) and (21.4)

$$
\frac{d}{d b} S_{R}=\frac{1+a b}{b} \sum_{n=0}^{\infty} n T_{n}-t S_{R}
$$

Now multiplying (4.2) and (4.3) by $R(1+a b)$ and $a b$ respectively and substituting into the left hand side of (4.1) gives

$$
(1+a b) t S_{R}-a(1+a b) \sum_{n=0}^{\infty} n T_{n}+a(1+a b) \sum_{n=0}^{\infty} n T_{n}-(1+a b) t S_{R}=0
$$

which is identical to the right hand side of (4.1) and the proof of Lemma 4.1 is complete.

ProOf of TheOREM 2.1: The proof of Theorem 2.1 will involve an induction argument on the parameter $R$. For the basis, $R=1$, a proof of (2.15) has been given by Cerone and Sofo [5] using Bürmann's Theorem. For $R=2$, a proof of (2.15), by Bürmann's Theorem has been given by Sofo and Cerone [16], and it was indicated that the same style of proof could be carried out for other integer values of $R$ (proof by exhaustion). For large integer values of $R$ the algebra for such a proof is horrendous, even for algebraic packages such as Macsyma, hence the following induction argument settles the proof of (2.15). The induction argument for the right hand side of (2.15) will involve the recurrence relation (4.1). From (4.1)

$$
\begin{aligned}
S_{R+1} & =\frac{1}{R(1+a b)}\left[t S_{R}-a b \frac{d}{d b} S_{R}\right] \\
& =\frac{1}{R(1+a b)}\left[\begin{array}{c}
t \sum_{k=0}^{R-1} \frac{t^{R-k-1}}{(R-k-1) !} Q_{R, k}(0) \\
-a b \sum_{k=0}^{R-1} \frac{t^{R-k-1}}{(R-k-1) !} \frac{d}{d b} Q_{R, k}(0)
\end{array}\right] \\
& =\frac{1}{R(1+a b)}\left[\begin{array}{c}
\frac{R t^{R}}{R !} Q_{R, 0}(0)+\sum_{k=1}^{R} \frac{(R-k) t^{R-k}}{(R-k) !} Q_{R, k}(0) \\
-a b \sum_{k=1}^{R} \frac{t^{R-k}}{(R-k) !} \frac{d}{d b} Q_{R, k-1}(0)
\end{array}\right]
\end{aligned}
$$


where the counter in the third term has been adjusted. Now on collecting terms in (4.4) we have that

$S_{R+1}=\frac{1}{R(1+a b)} \sum_{k=1}^{R}\left[(R-k) Q_{R, k}(0)-a b \frac{d}{d b} Q_{R, k-1}(0)\right] \frac{t^{R-k}}{(R-k) !}+\frac{t^{R}}{(1+a b) R !} Q_{R, 0}(0)$.

From Lemma 3.2, after adjusting the counter $k$,

$$
R(1+a b) Q_{R+1, k}(0)=(R-k) Q_{R, k}(0)-a b \frac{d}{d b} Q_{R, k-1}(0)
$$

so that by substituting (4.6) into the square bracket of (4.5) we have that

$$
\begin{aligned}
S_{R+1} & =\frac{t^{R}}{(1+a b) R !} Q_{R, 0}(0)+\sum_{k=1}^{R} \frac{t^{R-k}}{(R-k) !} Q_{R+1, k}(0) \\
& =\frac{t^{R}}{R !} Q_{1,0}(0) Q_{R, 0}(0)+\sum_{k=1}^{R} \frac{t^{R-k}}{(R-k) !} Q_{R+1, k}(0)
\end{aligned}
$$

where $Q_{1,0}(0)$ is identified in (3.1) or Table 1 . By the convolution nature of the $Q(0)$ terms we may write equation (4.7) as

$$
\begin{aligned}
S_{R+1} & =\frac{t^{R}}{R !} Q_{R+1,0}(0)+\sum_{k=1}^{R} \frac{t^{R-k}}{(R-k) !} Q_{R+1, k}(0) \\
& =\sum_{k=0}^{R} \frac{t^{R-k}}{(R-k) !} Q_{R+1, k}(0)
\end{aligned}
$$

which completes the proof of Theorem 2.1.

For completeness it is now worthwhile to briefly indicate a functional relationship for the infinite sum (2.14). From the left hand side of (2.15), let

$$
t=a \tau, \rho=R-1 \text { and } \gamma=a b e^{a b}
$$

then

$$
\sigma_{\rho}(\tau)=\sum_{n=0}^{\infty} \frac{(-\gamma)^{n}(\tau+n)^{n+\rho}}{n !}, \rho=0,1,2,3, \ldots
$$

and

$$
\sigma_{\rho}(\tau)+\gamma \sigma_{\rho}(\tau+1)=\tau \sigma_{\rho-1}(\tau)
$$

Pyke and Weinstock [14] gave a functional relationship of (4.8) for the case of $R=1$ only. Sofo and Cerone [16] have given a proof of the functional form (4.8) for the general case of integer $R$. 


\section{EXTENSION OF RESULTS}

The dynamical system (2.1) may take other functional values of the forcing terms $w(t)$, other than $\delta(t)$, such that consequent results of (2.15) may be extended. If, for example we let (other forms may also be considered)

$$
w(t)=\frac{e^{-b t} t^{m-1}}{(m-1) !}
$$

in the system (2.1), where $m$ is a positive integer, and following the procedure of Section 2 , we obtain

$$
\begin{aligned}
\sum_{n=0}^{\infty}\left(\begin{array}{c}
n+R-1 \\
n
\end{array}\right) \frac{b^{n} e^{-b(t-a n)}(t-a n)^{n+m+R-1}}{(n+m+R-1) !} \\
=\sum_{\mu=0}^{R-1} \frac{t^{R-\mu-1} Q_{R, \mu}(0)}{(R-\mu-1) !}+\sum_{\nu=0}^{m-1} \frac{t^{m-\nu-1}}{(m-\nu-1) !} P_{m, \nu}(-b) .
\end{aligned}
$$

In identity (5.1) we have that

$$
\begin{aligned}
\nu ! P_{m, \nu}(-b) & =\lim _{p \rightarrow-b}\left[\frac{d^{\nu}}{d p^{\nu}}\left\{(p+b)^{m} F(p)\right\}\right] ; \quad \nu=0,1,2,3, \ldots,(m-1), \\
\mu ! Q_{R, u}(0) & =\lim _{p \rightarrow 0}\left[\frac{d^{\mu}}{d p^{\mu}}\left\{p^{R} F(p)\right\}\right] ; \quad \mu=0,1,2,3, \ldots,(R-1)
\end{aligned}
$$

and

$$
F(p)=\frac{1}{(p+b)^{m}\left(p+b-b e^{-a p}\right)^{R}} .
$$

For $R=1$ and $m=2$ we have

$$
\sum_{n=0}^{\infty} \frac{b^{n} e^{-b(t-a n)}(t-a n)^{n+2}}{(n+2) !}=-\frac{e^{-b t}}{b e^{a b}}\left[t+\frac{1+a b e^{a b}}{b e^{a b}}\right]+\frac{1}{b^{2}(1+a b)}
$$

and for $R=2$ and $m=3$

$$
\begin{aligned}
\sum_{n=0}^{\infty}\left(\begin{array}{c}
n+1 \\
n
\end{array}\right) & \frac{b^{n} e^{-b(t-a n)}(t-a n)^{n+4}}{(n+4) !} \\
= & e^{-b t}\left[\frac{t^{2}}{2\left(b e^{a b}\right)^{2}}+\frac{2 t\left(1+a b e^{a b}\right)}{\left(b e^{a b}\right)^{3}}+\frac{2\left(a b e^{a b}\right)^{2}+6 a b e^{a b}+3}{\left(b e^{a b}\right)^{4}}\right] \\
& +\frac{t}{b^{3}(1+a b)^{2}}+\frac{a^{2}}{2 b^{2}(1+a b)^{3}}-\frac{3}{b^{4}(1+a b)^{2}} .
\end{aligned}
$$


In the degenerate case, for $a=0$, from (5.1) we obtain the impressive identity

$$
\begin{aligned}
\sum_{n=0}^{\infty}\left(\begin{array}{c}
n+R-1 \\
n
\end{array}\right) \frac{b^{n} e^{-b t} t^{n+m+R-1}}{(n+m+R-1) !}= & \frac{(-1)^{R}}{e^{b t}} \sum_{\nu-0}^{m-1} \frac{(R)_{\nu} t^{m-\nu-1}}{b^{R+\nu} \nu !(m-\nu-1) !} \\
& +\sum_{\mu=0}^{R-1} \frac{(-1)^{\mu}(m)_{\mu} t^{R-\mu-1}}{b^{m+\mu} \mu !(R-\mu-1) !}
\end{aligned}
$$

where

$$
\left.\begin{array}{c}
(x)_{0}=1 \\
(x)_{\rho}=x(x+1) \ldots(x+\rho-1)
\end{array}\right\}
$$

is known as Pochhammer's symbol. The identities (5.1) and (2.15) may be differentiated and integrated with respect to $t$ to produce more identities.

\section{Conclusion}

A method for the summation of infinite series has been described, the generation of an infinite sum was achieved by the application of Laplace transforms and the closed form representation was proved by an induction argument. We have generalised a result originally given by Euler and indicated a method for the generation of many other series. The method described in this paper may be easily extended to handle, for example, dynamical systems with multiple delays. In a forthcoming paper the authors will apply the techniques of this paper to generate infinite series that may be represented in closed form which depend on a multiple number of dominant zeros; we shall develop closed form representations of the sum

$$
\sum_{n=0}^{\infty}\left(\begin{array}{c}
n+R-1 \\
n
\end{array}\right) \frac{b^{n k} e^{-b(t-a n)}(t-a n)^{n k+m+R k-1}}{(n k+m+R k-1) !}
$$

for general parameter values $a, b, k, m, R$ and $t$.

\section{REFERENCES}

[1] R. Bellman and K.L. Cooke, Differential difference equations (Academic Press, New York, 1993).

[2] B.C. Berndt, Ramanujan's notebooks, Part 1 (Springer-Verlag, Berlin, Heidelberg, New York, 1985).

[3] L. Bruwier, 'Sur l'equation fonctionelle $y^{(n)}(x)+a_{1} y^{(n-1)}(x+c)+\ldots+a_{n-1} y^{\prime}(x+(n-1) c)$ $+a_{n} y(x+n c)=0^{\prime}$, in Comptes Rendus du Congres National des Sciences, Bruxelles, 1930, pp. 91-97.

[4] L. Bruwier, 'Sur une equation aux derivees et aux differences melees', Mathesis 47 (1933), 96-105. 
[5] P. Cerone and A. Sofo, 'Summing series arising from integrodifferential-difference equations', (V.U.T. Technical report 53), Math. 9 (1995).

[6] A.K. Erlang, 'Telefon-ventetider et stykke sandsynlighed-sregning', Mathematik Tidsskrift $B 31$ (1920), p. 25.

[7] L. Euler, 'De serie Lambertina plurimisque eius insignibus proprietatibus', Acta Acad. Sci Petropolitanae (1779), 29-51; II, (1783).

[8] L. Euler, 'Opera omnia', B.G. Teubner, Leipzig, Serie 16 (1921), 350-369.

[9] W. Feller, An introduction to probability theory and its applications (John Wiley and Sons, New York, 1971).

10] P. Hall, Introduction to the theory of coverage processes (John Wiley and Sons, New York, 1988).

11] G.H. Hardy, P.V. Seshu Aiyar and B.M. Wilson (Editors), Collected papers of Srinivasa Ramanujan (Chelsea Publishing Company, New York, 1962).

12] J.L.W.V. Jensen, 'Sur une identite d'Abel et sur d'autres formules analogues', Acta Math. 26 (1902), 307-313.

13] G. Pólya and G. Szegö, Aufgaben und Lehrsatze aus der Analysis (Springer-Verlag, Berlin, Dover, New York, 1945).

14] R. Pyke and R. Weistock, 'Special infinite series', Amer. Math. Monthly 67 (1960), 704.

15] W.L. Smith, 'Renewal theory and its ramifications', Royal Stat. Soc. J. Ser. B 20 (1958), 243-302.

16] A. Sofo and P. Cerone, 'Generalisation of a waiting time relation', J. Math. Anal. Appl. 214 (1997), 191-206.

17] H.C. Tijms, Stochastic modelling and analysis: $A$ computational approach (John Wiley and Sons, New York, 1986).

18] S.J.R. Woodward and G.C. Wake, 'A differential-delay model of pasture accumulation and loss in controlled grazing systems', Math. Biosci. 121 (1994), 37-60.

Department of Computer and Mathematical Sciences

Victoria University of Technology

PO Box 14428 MCMC

Melbourne Vic 8001

Australia 